CERN-TH.6918/93

\title{
ON NON-PERTURBATIVE EFFECTS AT THE HIGH-TEMPERATURE ELECTROWEAK PHASE TRANSITION
}

\author{
M. Shaposhnikov ${ }^{1}$ \\ Theory Division, CERN, \\ CH-1211 Geneva 23, Switzerland
}

\begin{abstract}
It is argued that confining effects in 3-dimensional non-Abelian gauge theories (hightemperature limit of 4-dimensional ones) imply the existence of the condensates of the gauge and Higgs fields in 3-d vacuum. This non-perturbative effect can decrease the energy of the phase with unbroken symmetry and may result in the creation of a barrier separating the broken and unbroken phases. Thus the high-temperature phase transitions in gauge theories can be stronger first order than is expected from perturbation theory. The applications of these results to electroweak baryogenesis are briefly discussed.
\end{abstract}

CERN-TH.6918/93

June 1993

\footnotetext{
${ }^{1}$ On leave of absence from the Institute for Nuclear Research of the Russian Academy of Sciences, Moscow 117312, Russia.
} 


\section{Introduction}

The study of the high-temperature phase transitions in the gauge theories [1]-[3] provides an interesting interplay between particle physics and cosmology. Recently considerable progress has been achieved in the understanding of the perturbative expansion for the effective potential of the scalar field at high temperatures [4]-[15]. One can summarize the present understanding as follows. Let us take for definiteness the standard electroweak theory with one scalar doublet. Then at zero temperatures the one-loop effective potential has the form

$$
V(\phi)_{T=0}=-(2 \lambda+B) \sigma^{2} \phi^{2}+\lambda \phi^{4}+B \phi^{4} \log \left(\phi^{2} / \sigma^{2}\right)
$$

where $\phi=\sigma=250 \mathrm{GeV}$ corresponds to the minimum $V(\phi)$,

$$
B=\frac{3}{64 \pi^{2}}\left(2 m_{W}^{4}+m_{Z}^{4}-4 m_{t}^{4}\right)
$$

Here all particle masses are given in terms of the vacuum expectation value of the Higgs field. The Higgs mass is given by $M_{H}^{2}=4 \sigma^{2}(3 B+2 \lambda)$. For $\lambda \ll g_{W}^{2}$ the main contribution to the finite-temperature effective potential comes from the gauge loops; the result of the standard calculation in the high-temperature limit ( $T$ is much larger than any mass scale) is $[4,2]$ :

$$
\Delta V_{T}=\frac{1}{8} T^{2} \phi^{2}\left(2 m_{W}^{2}+m_{Z}^{2}+2 m_{t}^{2}\right)-\frac{T \phi^{3}}{4 \pi}\left(2 m_{W}^{3}+m_{Z}^{3}\right) .
$$

The naive loop expansion of the effective potential works provided the mass of the gauge bosons induced by the Higgs mechanism, $M_{W}(\phi)=\frac{1}{2} g_{W} \phi$, is larger than the Debye screening scale $M_{D}=C_{D} g_{W} T$ (for the electroweak theory with three fermionic generations $C_{D}^{2}=\frac{11}{6}$ ). In order to go to the smaller values of $\phi$ one has to perform a resummation of the perturbation theory. This can be done with the use of gap equations for plasma masses taking into account the Debye screening [14, 13, 15]. Roughly speaking, the effect of the resummation reduces the coefficient in front of the

$\phi^{3}$ term by a factor $\frac{2}{3}$ [10] (see also [8, 9]), so that the first-order phase transition is weaker than one would expect from eq.(3). The effective potential with this effect taken into account is

$$
V_{T}(\phi)=\frac{1}{8}\left(T^{2}-T_{0}^{2}\right) \phi^{2}\left(2 m_{W}^{2}+m_{Z}^{2}+2 m_{t}^{2}\right)-\frac{2}{3} \frac{T \phi^{3}}{4 \pi}\left(2 m_{W}^{3}+m_{Z}^{3}\right)+\lambda \phi^{4},
$$

where

$$
T_{0}^{2}=\frac{8 \sigma^{2}(B+2 \lambda)}{2 m_{W}^{2}+m_{Z}^{2}+2 m_{t}^{2}} .
$$

It is argued that for sufficiently small Higgs masses (say, $m_{H}<70 \mathrm{GeV}$ ) the improved perturbative calculation of the effective potential is self-consistent if $M_{W}(\phi)>M_{M} \sim$ $\frac{1}{3 \pi} g_{W}^{2} T[14,13,15]$. Here $M_{M}$ is the so-called magnetic mass providing the screening of the static gauge fields in the plasma, which cures the infrared problem in the thermodynamics of Yang-Mills fields $[16,17]$. So, one expects that the first-order electroweak 
phase transition is rather weak for those Higgs masses. For the larger Higgs masses, the approximation breaks down, although there is an indication that the phase transition is actually of the second order [14].

Thus, the perturbative calculations of the effective potential seem to provide a selfconsistent picture of the phase transitions in gauge theories at sufficiently small scalar self-coupling constants. According to perturbation theory, the only requirement that has to be met is the smallness of the ratio $M_{M} / M_{W}(\phi) \sim \frac{2 g_{W} T}{3 \pi \phi}$. This value is indeed small for the interesting range of temperatures and fields $\phi$ for cosmological phase transitions in Grand Unified Theories and the electroweak theory, so that one might think that we achieved (an almost complete) understanding of those phenomena.

The aim of the present paper is to argue that non-perturbative effects can change the conclusions drawn from the study of the perturbative expansion of the effective potential. The paper is organized as follows. In Section 2 we show that 3-d nonperturbative effects are likely to decrease the free energy of the system in the unbroken phase, thus decreasing the value of the critical temperature. In Section 3 we discuss the contribution of some set of non-perturbative fluctuations to $V(\phi)$. In Section 4 we analyse the dynamics of the phase transition in cosmology and argue that the phase transition can be actually a very strongly of the first-order, contrary to the result obtained with perturbative calculation. Some applications of these results to cosmology and electroweak baryogenesis are discussed in Section 5. We summarize our results in the Conclusion.

\section{Non-perturbative effects in non-Abelian gauge theories at high temperatures}

In what follows we confine ourselves to the study of the phase transition in $\mathrm{SU}(2)$ gauge theory with one Higgs doublet, without fermions. For numerical presentations we will use the electroweak gauge coupling, $g_{W}=2 / 3$. The results can be easily extended to a more general case.

Our starting point is a dimensionally reduced gauge theory (see [18]-[22] and a recent discussion in [23]). The finite-temperature field theory is equivalent (for static quantities such as the effective potential we are interested in) to a Euclidean field theory, with compact fourth dimension whose size is $\frac{1}{T}$. Bosonic (fermionic) fields obey periodic (antiperiodic) boundary conditions in Euclidean time so that 'energies' of particles are discrete $\left(\omega_{B}=2 \pi n T, \omega_{F}=\pi(2 n+1) T, n=0, \pm 1, \pm 2, \ldots\right.$. If all mass scales of the theory are smaller than the temperature (this is true for the theory with small coupling constants in unbroken phase and for the system in the broken phase provided the Higgs-induced mass $M_{W}$ is small with respect to the temperature) all fermionic modes and non-zero bosonic modes can be integrated out. In this way one gets the effective 3 -d gauge theory with the action [23]:

$$
S_{3}=\int d^{3} x\left\{\frac{1}{4} F_{i j}^{a} F_{i j}^{a}+\frac{1}{2}\left(D_{i} A_{0}\right)^{a}\left(D_{i} A_{0}\right)^{a}+\left(D_{i} \phi\right)^{\dagger}\left(D_{i} \phi\right)+\right.
$$




$$
\begin{aligned}
& +\frac{1}{2} \frac{5}{6} g_{3}^{2} T A_{0}^{a} A_{0}^{a}+\frac{g_{3}^{4}}{12 \pi^{2}} \frac{17}{16}\left(A_{0}^{a} A_{0}^{a}\right)^{2}+ \\
& +\left[-\frac{1}{2} m_{H}^{2}+\left(\frac{3}{16} g_{3}^{2}+\frac{1}{2} \lambda_{3}\right) T\right] \phi^{\dagger} \phi+\lambda_{3}\left(\phi^{\dagger} \phi\right)^{2}+ \\
& \left.+\frac{1}{4} g_{3}^{2} A_{0}^{a} A_{0}^{a} \phi^{\dagger} \phi\right\}+ \text { counterterms, }
\end{aligned}
$$

containing, in addition to 3-d gauge fields and the Higgs doublet, a scalar field in adjoint representation (former $A_{0}$ component of the 4-d gauge field). There are also other higher polynomial contributions to this effective Lagrangian; they are, however, suppressed by the powers of temperature. In this equation $g_{3}^{2}=g_{W}^{2} T, \lambda_{3}=4 \lambda T$.

The 3-d effective Lagrangian contains four dimensionful parameters. The first one is just the Debye screening mass $\sim g_{W} T$; the second scale is a dimensionful coupling of the 3-d gauge theory $g_{3}^{2}=g_{W}^{2} T$; the third one is the mass of the Higgs $m(T)^{2}=$ $\left[-\frac{1}{2} m_{H}^{2}+\left(\frac{3}{16} g_{3}^{2}+\frac{1}{2} \lambda_{3}\right) T\right]$; and the fourth is the scalar self-coupling. The phase transition is expected to happen near $m^{2}(T)=0$, so that at this point there is a hierarchy of scales, $M_{D} \gg g_{3}^{2}, M_{D} \gg m(T)$. Hence, one can integrate out the $A_{0}$ field and study the action containing just the 3-d gauge fields and the Higgs field. From this exercise it is obvious how the expansion parameter $\frac{g_{3}}{2 \pi M_{W}(\phi)}$ arises when one computes gauge loops in the effective potential. Higher-order corrections generate some powers of $g_{3}$; one has to compensate these powers in order to get a correct dimension for the expression, and the only dimensionful quantity at hand is the mass of the $W$, originated from the Higgs mechanism. As usually in a loop expansion one gets a number of $2 \pi$ 's in the denominator from the integration over momenta.

In some cases, however, the region of applicability of the perturbation theory cannot be derived from the analysis of the perturbative expansion. The well-known example is provided by fermionic number non-conservation in the electroweak theory [24]. Here the amplitudes for the processes with B-violation are equal to zero in any order of perturbation theory while these processes do occur because of instantons. Another example, which is very close in spirit to the question we are discussing, is provided by QCD. Since we will use this analogy quite intensively, let us discuss in some detail the perturbation theory in QCD. As a classic example consider the ratio $R=\frac{\sigma\left(e^{+} e^{-} \rightarrow \text { hadrons }\right)}{\sigma\left(e^{+} e^{-} \rightarrow \mu^{+} \mu^{-}\right)}$ at some energy scale $s \sim 1 \mathrm{GeV}^{2}$ for three fermionic flavours. A first-order QCD correction to this ratio is known for a long time, $R=2\left[1+\frac{\alpha_{s}}{\pi}\right]$. The scale at which one should take $\alpha_{s}$ is not determined at this level. The computation of the three-loop corrections [25] allow one to fix the scale in $\alpha_{s}$ and one gets

$$
R=2\left[1+\frac{\alpha_{s}\left(s / \Lambda^{2}\right)}{\pi}+1.64\left(\frac{\alpha_{s}\left(s / \Lambda^{2}\right)}{\pi}\right)^{2}\right],
$$

where $\Lambda \sim 100-200 \mathrm{MeV}$ is the scale of the strong interactions in the $\overline{M S}$ scheme. The convergence of the perturbation theory is pretty good: at, say, $\sqrt{s} \sim 700 \mathrm{MeV}$, one has numerically $R=2[1+(0.12-0.18)+(0.024-0.05)]$ where the first number in brackets refers to $\Lambda=100 \mathrm{MeV}$ and the second one to $\Lambda=200 \mathrm{MeV}$. The natural 
tendency would then be to conclude that we know $R$ with quite a good precision (say, $5 \%$ ) at this energy scale. This conclusion is, however, evidently wrong as can be seen from the experimental data, since precisely at this scale one has $\rho$-meson resonance and the correct $R$-ratio has nothing in common with a perturbative expression.

The same story happens with other confining theories, such as $\mathrm{QCD}_{2}\left(N_{c}\right)$ in two dimensions [26]. Here, at least in the limit of an infinite number of colours $\left(N_{c} \rightarrow \infty\right)$ the spectrum of the states is discrete and there is no continuum in the spectrum, contrary to expectations from any given order of perturbation theory.

One of the manifestations of confinement is the existence of the non-perturbative condensates of the different composite operators. For example, in QCD there are condensates of quark and gluonic fields, $\langle(\bar{q} q)\rangle \sim-(250 \mathrm{MeV})^{3}$ and $\left\langle\frac{\alpha_{s}}{\pi} G^{2}\right\rangle \sim(330 \mathrm{MeV})^{4}$ [27], which appear also in $\mathrm{QCD}_{2}$ [28]. In QCD in 4-d one can even relate the properties of resonances to condensates via QCD sum rules [29]; the predictions are quite impressive for a number of hadronic channels [29]. The analysis of the QCD sum rules indicates that the actual range of applicability of the perturbation theory comes not from the analysis of the perturbative expansion but from non-perturbative corrections.

Now we come back to our 3-d theory. Non-Abelian gauge theory in three dimensions has a lot in common with 4-d QCD. Namely, both theories are confining (one has 'logarithmic' confinement in perturbation theory and linear confinement beyond it in $3 \mathrm{~d}$ ). Therefore, it is natural to assume that the mechanism for the mass gap generation is the same in both theories ${ }^{2}$. In particular, one should expect the existence of condensates of gauge and scalar fields in 3-d non-Abelian theory. (The gauge field condensate in a pure 3-d Yang-Mills theory has been discussed also in a recent paper by J.M. Cornwall [32] in a different context.) The lowest-order gauge-invariant operators are $F^{2}$ (we shall call it gluonic condensate, in analogy with QCD) and $\phi^{\dagger} \phi$ (scalar condensate). Just on dimensional grounds, near the point where $m^{2}(T)=0$ one gets

$$
\left\langle F_{i j}^{a} F_{i j}^{a}\right\rangle=A_{F} g_{3}^{6}, \quad\left\langle\phi^{\dagger} \phi\right\rangle=A_{S} g_{3}, \quad\left\langle\left(\phi^{\dagger} \phi\right)^{2}\right\rangle=A_{4} g_{3}^{2},
$$

where $A_{F}, A_{S}$ and $A_{4}$ are some dimensionless numbers. In 4-d QCD, the numerical values of condensates can be found from the analysis of the experimental data; in $\mathrm{QCD}_{2}$ from the exact 't Hooft solution [28]. Here we do not have this possibility ${ }^{3}$.

The knowledge of these condensates allows one to estimate the influence of nonperturbative effects on the critical temperature of the phase transition. At critical

\footnotetext{
${ }^{2}$ In refs. $[30,31]$ the $0^{++}$glueball mass has been estimated in pure gauge $\mathrm{SU}(2)$ theory in 3 dimensions on the lattice. The two papers give consistent results for the glueball mass; according to [30] $M_{G}=(1.7 \pm 0.4) g_{3}$ and according to [31] $M_{G}=(2.2 \pm 0.2) g_{3}$. One can see that, in full analogy with QCD, the scale associated with non-perturbative effects (3-d glueball mass) is numerically larger than the perturbative scale $g_{3} / 2 \pi$, by a factor $4 \pi \sim 10$ (of course, the parametric dependence is the same). This means that the region of applicability of the perturbation theory is actually smaller that can be expected from the analysis of the perturbative expansion.

${ }^{3}$ One can try to extract the value of the gluonic condensate from the analysis of QCD sum rules, given the lattice value for glueball mass in 3d. However, since QCD sum rules do not work in QCD [33] and $\mathrm{QCD}_{2}[26,34]$ for the channel with $0^{++}$quantum numbers, we do not attempt to do this here. Unfortunately, we were also unable to extract any information concerning the magnitude of the scalar condensate from the available lattice data.
} 
temperature the free energies of the broken and unbroken phases coincide; and now we have to take into account the non-perturbative shift of the energy densities due to the existence of the condensates in the broken and unbroken phases. This shift can be easily derived with the scaling arguments. Indeed, vacuum energy density $\epsilon_{\text {vac }}$ in the 3-dimensional theory given by the functional integral

$$
\exp \left(-\epsilon_{v a c} V\right)=\int d A_{i}(x) \exp \left(-S_{3}\right)
$$

where $V$ is the 3 -d volume and $S_{3}$ is the action given by eq. (6).

Rescaling the fields as $A_{i}=\sqrt{g_{3}} \bar{A}_{i}, \quad \phi=\sqrt{g_{3}} \bar{\phi}$, and differentiating eq. (9) with respect to $g_{3}, m(T)^{2}$ and $\lambda_{3}$ one gets for small $\lambda_{3}$ and for $m^{2}(T)$ close to zero ${ }^{4}$ :

$$
\epsilon_{v a c}=-\frac{1}{3}\left\langle\frac{1}{4} F_{i j}^{a} F_{i j}^{a}-2 m^{2}(T) \phi^{\dagger} \phi-\lambda_{3}\left(\phi^{\dagger} \phi\right)^{2}\right\rangle \simeq-\frac{1}{12}\left\langle F_{i j}^{a} F_{i j}^{a}\right\rangle,
$$

where we take into account the fact that $\epsilon_{v a c} \sim g_{3}^{3}$ and used the equations of motion for the scalar field. So, again in full analogy with QCD one has an energy shift proportional (with the minus sign) to gluonic condensate. This energy shift is an analogue of the bag constant in $\mathrm{QCD}^{5}$. Note that eq. (10) is true in the broken phase minimum as well. The $\phi^{3}$ term in the effective potential in this phase arises from the perturbative value of $\left\langle F_{i j}^{a} F_{i j}^{a}\right\rangle$.

Clearly, non-perturbative contributions to the energy density should change the dynamics of the phase transitions at high temperatures. If the gluonic condensate is positive (as in Euclidean $\mathrm{QCD}_{4}[27]$ and $\mathrm{QCD}_{2}$ [28]), then non-perturbative effects 'dig' a 'pit' near the origin, therefore reducing the value of the critical temperature. For the negative gluonic condensate ${ }^{6}$ (this situation seems to be quite improbable, though) the value of the critical temperature is larger than that predicted by perturbation theory.

The change of the critical temperature can be estimated by using the value of the free energy of the system in the broken phase. It is natural to expect that in the broken phase, with non-zero vacuum expectation value of the Higgs field, non-trivial fluctuations giving rise to the gluonic condensate are highly suppressed (we will discuss this point in more detail in the next section), so that one should take into account this shift in the unbroken phase only. The critical temperature $T_{c}$ is then determined by the equation

$$
\epsilon_{v a c}=V_{T}(\phi),
$$

where $\frac{d V_{T}(\phi)}{d \phi}=0$. The shift depends on the mass of the Higgs boson and constant $A_{F}$. We present this dependence for positive $A_{F}$ in Fig. 1. One can see that at $M_{H}>50$ $\mathrm{GeV}$ and, say, $A_{F}=0.1$, the critical temperature is even lower than the temperature $T_{0}$, which is thought to be the temperature of absolute instability of the unbroken phase.

\footnotetext{
${ }^{4}$ For small $m^{2}(T)$ and $\lambda_{3}$ the contribution of the scalar condensate is small with respect to the contribution of the gluonic condensate.

${ }^{5}$ Note that the contribution of the gluonic condensate to the free energy is of the order of $g_{W}^{6} T^{4}$, precisely the term that is not computable by perturbation theory.

${ }^{6}$ In principle, the gluonic condensate (as well as the scalar condensate) can have either sign, since the positive-definiteness of the operator $F^{2}$ can be spoiled by renormalization.
} 
Numerically this shift is quite small for the light Higgs and gets larger for higher Higgs masses. It is clear that the changing of the effective potential at the origin generates also a barrier separating the broken and unbroken phases. In cosmology this barrier will prevent the phase transition to occur at the critical temperature and it happens at temperatures smaller than $T_{c}$. In the next section an attempt is made to estimate this effect.

\section{Estimate of the gluonic condensate}

We are mainly interested in the non-perturbative contributions to the effective potential at small $\phi$. It is clear that there is no hope to determine it in some regular way due to strong coupling and confinement in 3-d gauge theory. In some models, however, one can study this question by semi-classical methods in a small coupling regime. The example is provided by the Georgi-Glashow model in 3-d ( $\mathrm{SU}(2)$ gauge theory with a triplet of scalar fields and spontaneous symmetry breaking). It has been shown [35] that in this model confinement is due to instantons (coinciding with monopoles in this case). The shift of the vacuum energy density is just

$$
\epsilon_{v a c}=-\frac{M_{W}^{\frac{7}{2}}}{g_{3}} \alpha\left(\lambda / g_{W}^{2}\right) \exp \left(-\frac{4 \pi M_{W}}{g_{3}^{2}} \beta\left(\lambda / g_{W}^{2}\right)\right),
$$

where $\alpha$ and $\beta$ are some functions that can be numerically calculated, $\beta(0)=1, M_{W}$ is the $W$-mass. The semi-classical approximation does not work at small $M_{W}$, but one can see that this shift is negative and exponentially small in the broken phase and gets larger with the decrease of the vacuum expectation value.

The theory with the doublet of the scalar field is more complicated, since there are no stable solutions to the classical equations of motion even in the broken phase. So, one cannot perform the analysis in a semi-classical approximation. To get some insight into the problem, let us study the non-perturbative gauge field configurations, which may give rise to the gluonic condensate. We start from a pure Yang-Mills theory. The gluonic condensate is given by the functional integral

$$
\left\langle F_{i j}^{a} F_{i j}^{a}\right\rangle=\int d A_{i}(x) F_{i j}^{a} F_{i j}^{a} \exp (-S) .
$$

According to a suggestion made in [17] one can consider the contribution to eq. (13) of unstable monopole configurations characterized by some scale $\rho$. To be more specific, we choose as a trial configuration the 4 -d instanton at zero time (see also [36]),

$$
A_{i}^{a}(x)=\frac{1}{g_{3}} \epsilon_{a i j} \frac{x_{j}}{x^{2}+\rho^{2}} .
$$

The action of this configuration is

$$
S=\frac{3 \pi^{2}}{g_{3}^{2} \rho}
$$


There is a number of zero modes associated with this configuration, namely three translational zero modes and three rotational ones. The normalization factors associated with them can be computed in full analogy with the sphaleron zero-mode problem [37]. For rotational zero modes we have

$$
N_{\text {rot }}=8 \pi^{2}\left[\frac{8 \pi \rho}{3 g_{3}^{2}}\right]^{\frac{3}{2}} .
$$

Translational zero modes give the contribution

$$
N_{t r}=\left[\frac{16}{3 g_{3}^{2} \rho}\left(15 \pi / 8-\pi^{3} / 12\right)\right]^{\frac{3}{2}} .
$$

In order to compute the functional integral in the vicinity of this configuration, one should also introduce a normalization for a scale mode associated with $\rho$. It is given by

$$
N_{\rho}=\left[\frac{\pi}{2 g_{3}^{2} \rho}\right]^{\frac{1}{2}} .
$$

For dimensional reasons, the determinant of non-zero modes is just

$$
D=\kappa \frac{1}{\rho^{6}}
$$

where $\kappa$ is some dimensionless number. From the experience with the sphaleron [38], one would expect $\kappa \sim 1$.

Combining all factors together we obtain

$$
\left\langle F_{i j}^{a} F_{i j}^{a}\right\rangle=\int \frac{d \rho}{\rho} N_{\text {rot }} N_{t r} N_{\rho} D \frac{12 \pi^{2}}{g_{3}^{2} \rho} \exp \left(-\frac{3 \pi^{2}}{g_{3} \rho}\right)=0.36 \kappa g_{3}^{6} \sim 0.4 g_{3}^{6} .
$$

The integral over the scale converges at small $\rho$, owing to an exponential factor (the energy of the configuration diverges when $\rho \rightarrow \infty)$ and also converges at small $\rho$ due to a pre-exponential entropy factor. The scale at which the integral is saturated is about $\rho^{-1} \sim 2 g_{3}^{2} / \pi^{2}$. Of course, this estimate of the condensate is rather crude and it is impossible to compute corrections to it. For our future estimates, we consider the factor $A_{F}$ as a parameter of order $1^{7}$

Now, we can turn to our theory with Higgs fields. The presence of the scalar condensate due to the symmetry breaking suppresses the fluctuations of the gauge field. Again, one can take 't Hooft solution [24] for the scalar field in the vicinity of the instanton with boundary conditions $\phi(x \rightarrow \infty)=v$

$$
\phi(x)=\frac{\sigma_{i} x_{i}}{\left(x^{2}+\rho^{2}\right)^{\frac{1}{2}}}(0, v)
$$

\footnotetext{
${ }^{7} \mathrm{~A}$ very different estimate of the gluonic condensate can be found in [32], $A_{F} \sim 5 \cdot 10^{-3}$. Cornwall also concluded that non-perturbative effects introduce a negative energy shift in a pure Yang-Mills theory in 3-d.
} 
and repeat the previous calculation. In the limit of $m^{2}(T)=0$ and of a small scalar self-coupling constant, one obtains

$$
\left\langle F_{i j}^{a} F_{i j}^{a}\right\rangle(\phi)=A_{F} \frac{g_{3}^{6}}{\Gamma\left(\frac{15}{2}\right) 2^{\frac{13}{2}}} z^{\frac{15}{2}} K_{\frac{15}{2}}(z),
$$

where $K$ is a modified Bessel function and

$$
z=\frac{3 \pi^{2} v}{\sqrt{2} g_{3}} .
$$

As expected, the non-perturbative contribution decreases exponentially with the field $\phi$, so that non-perturbative effects are frozen in the broken phase. Again, it is difficult to estimate the corrections to this calculation; however, the non-perturbative contribution coming from the gluon condensate has the properties expected from the physical grounds, namely we are getting a negative energy shift at the origin, and this shift exponentially decreases in the broken phase.

To summarize, the effective potential for the scalar field, with the contribution of the non-perturbative effects and for small $\lambda$ and $m^{2}(T)$, is likely to have an approximate form

$$
V_{t o t}=V_{T}(\phi)-\frac{T}{12}\left\langle F_{i j}^{a} F_{i j}^{a}\right\rangle(\phi)
$$

This potential is shown in Fig. 2 at different values of the temperature, for $M_{H}=90$ $\mathrm{GeV}$ and $A_{F}=0.36$, as a function of $z$ and in Fig. 3 for $T=140 \mathrm{GeV}$ for small values of $z$. With this potential the temperature $T_{a}$ of the absolute instability of the unbroken phase is given by

$$
T_{a}^{2}=\frac{T_{0}^{2}}{1+\frac{16}{13} \pi^{2} g_{W}^{2} A_{F}}
$$

and is smaller than $T_{0}$.

\section{Dynamics of the phase transition}

With potential (24) one can study the dynamics of the phase transition. The crucial question here is the computation of the free energy of the nucleating bubbles of the new phase. The standard procedure is to find an extremum of the effective action for the scalar field ${ }^{8}$

$$
S_{e f f}=\int d^{3} x\left(\frac{1}{2}\left(\partial_{i} \phi\right)^{2}+V_{t o t}\right)
$$

with boundary conditions $\phi(x) \rightarrow 0, x \rightarrow \infty$, and $d \phi / d x=0$ at $x=0$. The phase transition is complete at the temperature $T_{*}$ at which the action for the bubble is about [39]

$$
4 \log \left(\frac{M_{P l}}{T}\right) \sim 160
$$

\footnotetext{
${ }^{8}$ The non-perturbative effects can also modify the kinetic term for the Higgs field in addition to the modification of the potential. We do not attempt to estimate the influence of this effect here.
} 
for the electroweak theory. As usual, $M_{P l}$ is the Planck mass. The problem can be solved numerically. The phase transition occurs at the temperature $T_{*}$, smaller than the critical temperature $T_{c}$. For example, at $A_{F}=1(0.36,0.12)$ and $M_{H}=130(90,60)$ $\mathrm{GeV}$, the actual temperature of the phase transition is $T_{*} \simeq 145(138,120) \mathrm{GeV}$. The corresponding values of $T_{0}$ are equal to 320 (220, 150) GeV. The jump of the order parameter is quite substantial. For all these examples $\delta \phi \sim 1.6 T$. So, non-perturbative effects seem to change the behaviour of the effective potential at the origin and convert a weakly first-order phase transition to a strongly first-order one, provided $A_{F}$ is not numerically small.

Of course, there are many uncertainties in this computation. The main one is associated with the fact that we are dealing with a confining theory and work actually in a strong coupling regime. The accuracy of the dimensional reduction with experimental $g_{W}$ is quite bad, since there is no hierarchy of scales $M_{M} \ll M_{D} \ll T$, which allow 4-d dynamics to be separated from a 3-d one. Numerically, all the scales are just the same and $\sim T$. It is not clear how this situation should be dealt with. Even inside our approach, at $T=T_{*}, m^{2}(T)$ is not small (remember that we neglected it in a previous computation), and the value of the scalar self-coupling is of the order of $g_{W}^{2}$. We do not think, however that the accounting for the $m^{2}$ and $\lambda$ can qualitatively change the conclusion that non-perturbative effects can make the phase transition strongly first order ${ }^{9}$.

Some support for this observation comes from the 4-d [40] and 3-d [23] lattice simulation of the electroweak phase transition. In particular, the data in [23] indicate that the actual temperature of the electroweak phase transition is lower than the one predicted by perturbation theory and that the actual region of metastability is larger. From [40] one sees that the actual jump of the order parameter is also larger than the one from perturbation theory.

\section{Applications to the electroweak baryogenesis}

All electroweak baryogenesis mechanisms require a first-order phase transition. Quantatively, the sphaleron mass in the broken phase must be sufficiently large [6],

$$
M_{s p h}(T) / T \simeq z_{v a c}>45
$$

in order for the baryonic asymmetry produced at the electroweak transition to survive to the present time. Here $z_{v a c}$ is the expectation value of the variable $z$ defined by (23). This requirement puts an upper bound on the Higgs mass. The analysis of the one-loop perturbation theory gives an upper bound $45 \mathrm{GeV}$ [6]. As is argued above, non-perturbative effects change the nature of the phase transition. With the form of the effective potential advocated in this paper, the critical mass of the Higgs boson

\footnotetext{
${ }^{9}$ The phase transition is stronger first order if $\epsilon_{v a c}\left(T_{0}\right)<0$ in the unbroken phase (see eq. (10)). If $\left\langle\left(\phi^{\dagger} \phi\right)^{2}\right\rangle<0$, this is always true. If the scalar condensate $\left\langle\left(\phi^{\dagger} \phi\right)^{2}\right\rangle>0$ and large enough, then the transition may be more weakly first order than in perturbation theory for a sufficiently heavy Higgs boson, $\lambda_{3}>\frac{\left\langle F^{2}\right\rangle}{4\left\langle\left(\phi^{\dagger} \phi\right)^{2}\right\rangle}$.
} 
may be much larger and depends on the unknown parameter $A_{F}$. For example, for $A_{F}=1(0.36,0.12), M_{H}^{\text {crit }} \simeq 130(90,60) \mathrm{GeV}$. While the uncertainties in these estimates may be large, it seems clear that non-perturbative effects may considerably increase $M_{H}^{c r i t}$. In other words, electroweak baryogenesis can be possible with the experimentally allowed Higgs. At the same time, the cosmological upper bound on the Higgs mass is still much stronger than the one originating from triviality arguments. To establish the cosmological bound with a high accuracy, the better understanding of the non-perturbative effects in 3-d theories is required. The lattice simulations can clarify the question. However, as shown in [23] the lattice size should be huge in order to take into account all relevant effects.

Another important parameter for the electroweak baryogenesis is the thickness of the domain wall $a$. With the effective potential derived with the use of perturbation theory, one gets quite a thick wall. Say, with $M_{H}=35 \mathrm{GeV}$ at the transition point, $a \sim 40 / T$. This value is larger than the mean free path of the quarks in the plasma, which is estimated to be $l \sim 4 / T$ [10]. With our effective potential, the situation is different. The domain-wall thickness is roughly the inverse mass of the Higgs boson in the broken phase at $T=T_{*}$,

$$
a \sim \frac{1}{g_{w} T} \sqrt{\frac{8 T_{*}^{2}}{3\left(T_{0}^{2}-T_{*}^{2}\right)}} \sim \frac{1-3}{T}
$$

for the critical value of the Higgs mass and $A_{F}=(1-0.1)$. This satisfies the assumptions, made in the computation of the baryonic asymmetry of the Universe in the standard electroweak theory $[8,41]$. To conclude, the minimal standard model electroweak baryogenesis seems to be in good shape.

The non-perturbative effects in 3-d theory discussed in this paper may change the dynamics of the grand unified phase transitions. In particular, the amount of supercooling is expected to be larger than follows from the perturbation theory. This may result in a prolongation of the inflation period. The study of these questions is beyond the scope of this paper.

\section{Conclusion}

We have argued that the non-perturbative effects in $3 \mathrm{~d}$ gauge theories associated with confinement can change the dynamics of the high temperature phase transitions in the gauge theories. These effects very likely generate the condensates of different fields, like gluonic condensate, which decrease the energy of the vacuum in the unbroken phase. This change results in the decrease of the critical temperature of the phase transitions and create an additional barrier separating the broken and unbroken phases. For the standard electroweak model non-perturbative effects can make the phase transition to be strongly first order and make an electroweak baryogenesis possible for the experimentally allowed Higgs boson.

The author is deeply indebted to K. Kajantie, K. Rummukainen and A. Zhitnisky for many valuable disscussions and suggestions. He is also grateful to K. Farakos, G. 
Farrar, A. Kataev and I. Tkachev for helpful comments.

\section{References}

[1] D.A. Kirzhnitz, JETP Lett. 15 (1972) 529;

D.A. Kirzhnitz and A.D. Linde, Phys. Lett. 72B (1972) 471

[2] L. Dolan and R. Jackiw, Phys.Rev. D9 (1974)3320

[3] S. Weinberg, Phys.Rev. D9 (1974) 3357

[4] D.A. Kirzhnitz and A.D. Linde, Ann. Phys. 101 (1976) 195;

A.D. Linde, Nucl. Phys. B216 (1983) 421, Rep. Prog. Phys. 47 (1984)925

[5] K. Takahashi, Phys. Rev. Lett. 56 (1986) 7

[6] M.E. Shaposhnikov. Nucl. Phys. B287 (1987) 757; A.I. Bochkarev and M.E. Shaposhnikov, Mod. Phys. Lett. 2A (1987) 417

[7] G.W. Anderson and L.J. Hall, Phys. Rev. D45 (1992) 2685

[8] M.E. Shaposhnikov, Phys. Lett. B277 (1992)324; B282 (1992) 483(E);

[9] M. Carrington, Phys. Rev. D45 (1992) 2933

[10] M. Dine, R.G. Leigh, P. Huet, A. Linde and D. Linde, Phys. Rev. D46 (1992) 550

[11] K. Enqvist, J. Ignatius, K. Kajantie and K. Rummukainen, Phys. Rev. D45 (1992) 3415

[12] D.E. Brahm, C.G. Boyd and S.D.H Hsu, Preprint EFI-92-22, 1992

[13] P. Arnold and E. Espinosa, Preprint UW/PT-92-18, 1992

[14] M. Quiros, J.R. Espinosa and F. Zwirner, Preprint CERN-TH.6577/92, 1992

[15] W. Buchmüller, Z. Fodor, T. Helbig and D. Walliser, Preprint DESY 93-021, 1993

[16] A.D. Linde, Phys. Lett. 96B (1980) 289;

[17] D. Gross, R. Pisarski and L. Yaffe, Rev. Mod. Phys. 53 (1981) 43

[18] R. Jackiw and S. Templeton, Phys. Rev. D23 (1981) 2291

[19] T. Appelquist and R.D. Pisarski, Phys. Rev. D23 (1981) 2305

[20] S. Nadkarni, Phys. Rev. D27 (1983) 917 and D38 (1988) 3287

[21] P. Ginsparg, Nucl. Phys. B170 (1980) 388 
[22] N.P. Landsman, Nucl. Phys. B322 (1989) 498

[23] K. Kajantie, K. Rummukainen and M. Shaposhnikov, CERN preprint CERNTH.6901/93, 1993

[24] G. 't Hooft, Phys. Rev. Lett. 37 (1976) 8; Phys.Rev. D14 (1976)3432.

[25] K.G. Chetyrkin, A.L. Kataev and F.V. Tkachov, Phys. Lett. B85 (1979) 277;

M. Dine and J. Sapirstein, Phys. Rev. Lett. 43 (1979) 668;

S.G. Gorishny, A.L. Kataev and S.A. Larin, Phys. Lett. 212B (1988) 238

[26] G.'t Hooft, Nucl. Phys. B75 (1974) 461

[27] A. Vainshtein, V. Zakharov and M. Shifman, JETP Lett. 27 (1978) 55.

[28] A.R. Zhitnitski, Sov. J. Nucl. Phys. 43 (1986) 999; Phys. Lett. B165 (1985) 405

[29] V. Novikov, M. Shifman, A. Vainshtein and V. Zakharov, Nucl. Phys. B147 (1979) $385,448,519$

[30] A. Irbäck and C. Peterson, Phys. Lett. 174B (1986) 99

[31] G. Koutsoumbas, K. Farakos and S. Sarantakos, Phys. Lett. 189B (1986) 173

[32] J.M. Cornwall, Preprint UCLA/92/TEP/51, 1992

[33] V. Novikov, M. Shifman, A. Vainshtein and V. Zakharov, Nucl. Phys. B191 (1981) 301

[34] A.R. Zhitnitski, Sov. J. Nucl. Phys. 44 (1986) 139

[35] A.M. Polyakov, Nucl. Phys. B120 (1977) 429

[36] V.A. Rubakov, Nucl. Phys. B256 (1985) 434.

[37] P. Arnold and L. McLerran, Phys.Rev. D36 (1987) 581

[38] L.Carson, Xu Li, L. McLerran and R.T. Wang, Phys. Rev. D42 (1990) 2127

[39] A.D. Linde, Nucl.Phys. B216 (1983) 421.

[40] B. Bunk, E.-M. Ilgenfritz, J. Kripfganz, A. Schiller, Phys. Lett. B284 (1992) 371;

Bielefeld Preprint BI-TP 92/46

[41] G.R. Farrar and M.E. Shaposhnikov, Phys. Rev. Lett. 70 (1993) 2833; Preprint CERN-TH.6734/RU-93-11, 1993 
Figure 1: The dependence of the quantity $\frac{T_{c}-T_{0}}{T_{0}}$ on the mass of the Higgs boson for different values of the gluonic condensate. Curves $1,2,3$ correspond to $A_{F}=0,0.12,0.36$.

Figure 2: Effective potential as a function of $z$ for $M_{H}=90 \mathrm{GeV}$ and $A_{F}=0.36$ for different temperatures. Curve $1-T=220 \mathrm{GeV}, 2-199 \mathrm{GeV}, 3-190 \mathrm{Gev}, 4-150$ $\mathrm{GeV}$.

Figure 3: Effective potential as a function of $z$ for $M_{H}=90 \mathrm{GeV}$ and $A_{F}=0.36$ for $T=140 \mathrm{GeV}$ at small $z$. 